\title{
A generic crystallopathic model for chronic kidney disease progression
}

\author{
Orson W. Moe \\ Charles and Jane Pak Center for Mineral Metabolism and Clinical Research, Department of Internal Medicine, Division of Nephrology, Department of Physiology, University of Texas Southwestern Medical \\ Center, Dallas, Texas, USA.
}

\begin{abstract}
Chronic kidney disease (CKD) has reached epidemic proportions globally. The natural course of chronic kidney disease is almost uniformly progressive, albeit at different rates in different individuals. The downhill course appears to pervade kidney diseases of all etiologies and seems to spiral down a selfperpetuating vortex, even if the original insult is ameliorated or controlled. In this issue of the $\mathrm{JCl}$, Shiizaki, Tsubouchi, and colleagues proposed a model of renal tubule luminal calcium phosphate crystallopathy that accounts for renal function demise. Calcium phosphate crystals attached to TLR4 and underwent endocytosis at the brush border, triggering inflammation and fibrosis. This mechanism might operate in different kinds of kidney disease, with a theoretical phosphate concentration threshold in the proximal tubular lumen, beyond which is triggered undesirable downstream effects that eventuate in loss of renal function. If this model parallels human CKD, clinicians may focus efforts on determining phosphate exposure in the proximal tubular lumen.
\end{abstract}

\section{Chronic kidney disease progression is universal}

Chronic kidney disease (CKD) has reached epidemic proportions globally, imparting dire health and economic burdens on humankind. The progression of CKD appears to be nearly universal, regardless of the underlying etiology, strongly indicating that there are common underlying pathogenic mechanisms that drive the downward spiral of CKD from diverse causes. The prevalence of patients with stage 3 CKD far exceeds that of patients with more advanced stage 4 and 5 CKD (1). One would prefer to believe that a substantial fraction of patients with stage $3 \mathrm{CKD}$ are stable without progression, but the sobering and disturbing fact is that stage $3 \mathrm{CKD}$ has a high mortality rate. It is more likely that a patient with stage 3 CKD will die before reaching endstage kidney disease.

\section{Concept of crystallopathy}

A crystal is a structurally ordered geometric solid that can be organic or inorganic and can form in living tissues and body fluids. The concept of crystallopathy has been long established. Crystallopathy can be defined broadly as a state in which crystals and crystal-like particles directly cause or contribute to undesirable biologic consequences. The negative impact of the crystals distinguishes crystallopathy from physiologic crystal formation, such as ossification of calcified tissues in mammals

Related Article: https://doi.org/10.1172/JCl145693

Conflict of interest: OWM is a co-inventor of effervescent calcium magnesium citrate; potassium magnesium citrate synthetic anti-Klotho antibodies; and inhalational delivery using nanoparticles containing the erythropoietin receptor (patent no. 10,251,909 B2: Potassium-magnesium citrate as a surrogate of the DASH diet in managing hypertension; patent no. 10,092,593 B2: Citrate-rich calcium-magnesium supplement and uses thereof; patent no. 10912791: Compositions comprising antisense-encoded erythropoietin receptor and use thereof.)

Copyright: @ 2021, American Society for Clinical Investigation.

Reference information: / Clin Invest. 2021;131(16):e151858. https://doi.org/10.1172/JCI151858.

or the water-conserving tactic of nitrogen excretion in uricotelic organisms.

Attempts have been made to classify these conditions in the kidney and elsewhere on the basis of the type of crystals (e.g., uric acid, calcium phosphate, cholesterol), their anatomic locale (e.g., interstitium, tubule lumina), and the downstream pathobiologic effects (e.g., inflammation, fibrosis, obstruction) (2). Although this classification system is a good organizing tool conceptually, frequent overlaps render a strict classification difficult and underscore the importance of understanding the pathophysiology of each condition.

\section{Intraluminal calcium phosphate crystals and pathogenesis in CKD progression}

Shiizaki, Tsubouchi, and coworkers, under the leadership of Makoto Kuro-o, present a model in which solid-phase calcium phosphate particles in the lumen of the renal tubules activate a cascade that culminates in tissue damage and progression of CKD (Figure 1) (3).

These calcium phosphate crystals appear to differ from calciprotein particles (CPPs), which are complex organized particles with inorganic and organic constituents found in the blood at high concentrations in CKD (4) and are cleared by scavenger receptor A (5). The study by Shiizaki, Tsubouchi, and colleagues (3) found no evidence that CPPs are filtered from the blood, but rather that calcium phosphate precipitates in situ in the proximal tubule lumen. The TLR4 is necessary for the genesis of the pathology and is proposed to be the receptor that fulfills multiple functions of holding the calcium phosphate crystal in place on the proximal tubule brush border and activating p38/ $\mathrm{NF}-\kappa \mathrm{B}$ signaling, endocytosis, and nonconventional signaling pathways (3) - all or some of which result in tubular fibrosis and interstitial inflammation. One curious 


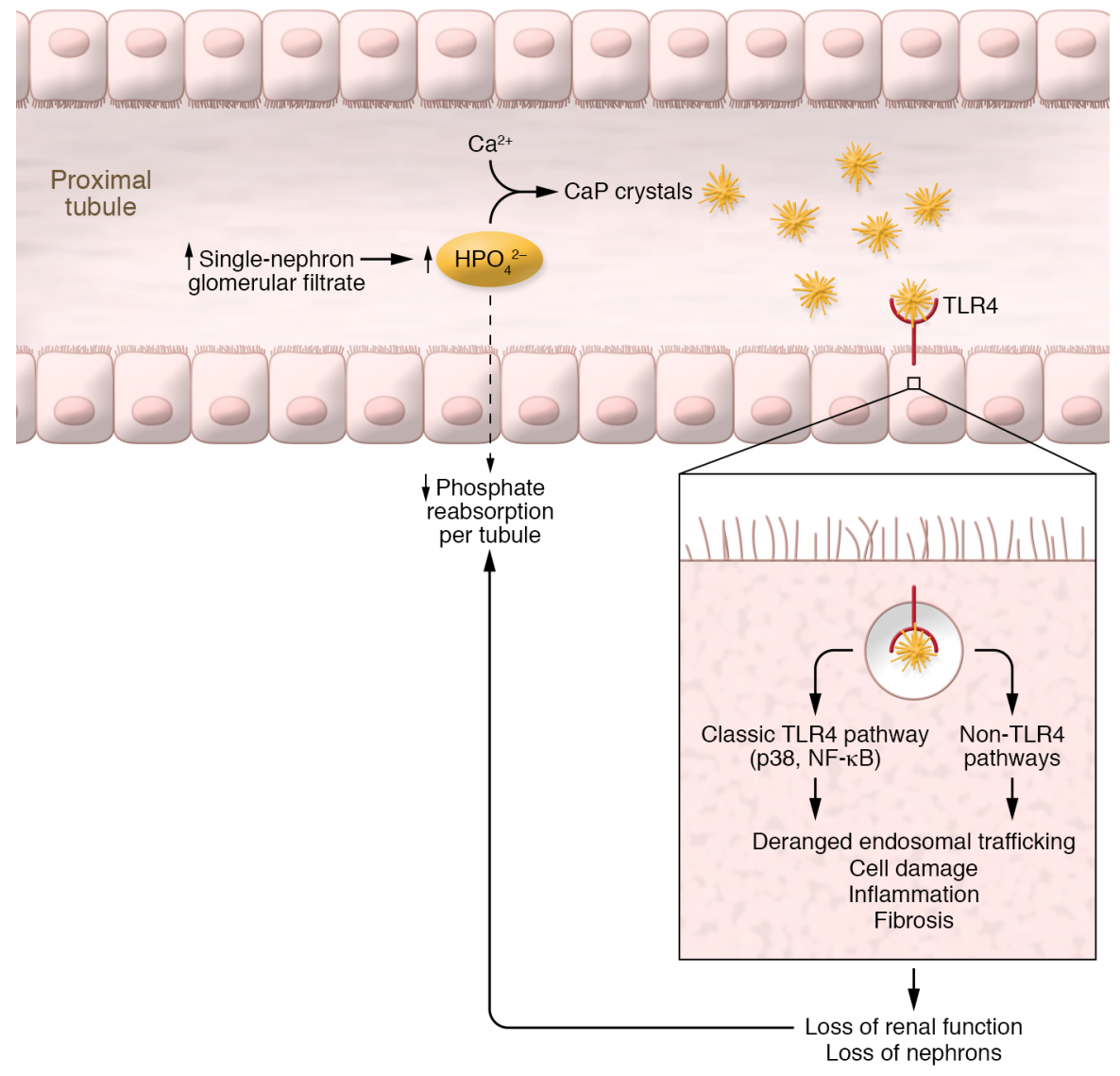

Figure 1. Model of renal tubular crystallopathy and CKD progression proposed by Shiizaki, Tsubouchi, and coworkers. In CKD of any etiology, high filtration in intact nephrons and reduced phosphate reabsorption driven by high parathyroid hormone and FGF23, and CKD itself, increase luminal phosphate concentrations. When calcium phosphate reaches a certain threshold in the end of the proximal tubule, crystals form and bind to brush border TLR4, which secures the crystals on the cell surface, internalizes the crystals, and activates a series of cascades. Subsequent damage of the tubules sets off inflammation and fibrosis in the tubulointerstitium, engendering further loss of renal function. The process reiterates in a vicious cycle.

finding is that when damage, inflammatory, and fibrotic biomarkers were quantified as a function of the estimated end-proximal tubule phosphate concentration, the response was rather flat until an inflection point resulted in a linear increase and positive association. This response created the concept of a threshold, below which lies tolerance and resilience and above which disease develops.

A prevalent axiom in CKD pathobiology is the self-perpetuating nature of CKD. While there is some truth to the notion that loss of nephrons begets more loss of nephrons, it is also well known that in humans the same primary disease can affect individuals with the same measurable severity yet progress at widely disparate rates. There must exist driving forces that govern progression.
The paradigm presented by Shiizaki, Tsubouchi, and coworkers (3) does not contradict the various existing models of generic CKD progression but is likely harmonious with and complementary to prior models.

In 1960, Bricker and colleagues put forth the intact nephron hypothesis (6) based on several dog models of renal disease. This hypothesis states that in a diseased kidney, the functional intact nephrons adapt and compensate for the diminished renal function due to loss of nephrons. Bricker and colleagues discussed in particular the changes in phosphate handling, indicating that despite the lower filtered phosphate load (low glomerular filtration rate [GFR]), tubular reabsorption must decrease to achieve the appropriate level of phosphate excre- tion $(6,7)$. What was not considered in the Bricker et al. model was the consequence of the high phosphate load at the end of the proximal tubule due to a high single-nephron filtration rate and the adaptive decreased proximal tubule phosphate reabsorption that result in flooding of the end of the proximal tubule and the rest of the nephron with high luminal phosphate concentrations.

Meyer, Anderson, and Brenner presented intraglomerular hypertension begetting glomerular damage, which manifests as sclerosis, as a universal model of progressive functional decline (8). The emphasis was placed on the hydraulic damage imposed by the intraglomerular hypertension that accompanies hyperfiltration. Indeed, maneuvers that alleviate the high glomerular pressure also retard the progression of kidney disease (9). However, contemporaneous with lower glomerular pressure is a lower single-nephron filtration rate. One can shift the primary lesion from glomerular hypertension to hyperfiltration. A lower filtered load per nephron will deliver less phosphate to form calcium phosphate crystals and trigger less severe crystallopathy.

The atubular glomerulus was first described in the 1930s by Jean Oliver, who noted that some glomeruli appeared to be anatomically disconnected from the proximal tubule (10). Interestingly, the damaging effects of phosphate feeding were highlighted by McKay and Oliver around the same time (11). The tubular damage in turn leads to secondary glomerular damage and loss of renal function via different mechanisms in a wide range of models of chronic renal damage $(12,13)$. Luminal calcium phosphate crystal formation is one mechanism that can lead to chronic, relentless loss of renal function.

\section{Therapeutic opportunities}

The ultimate goal of elucidating pathophysiology is to provide diagnostic and therapeutic opportunities. If the instigating event is calcium phosphate crystallopathy in the proximal tubule lumen, the primary goal will be to reduce the amount of phosphate reaching the proximal tubule lumen. Increased phosphate excretion per nephron is important to maintain an external balance and prevent systemic phosphotoxicity, but the cost is the highly unde- 
sirable consequence of urinary luminal crystallopathy. The logical maneuver is to decrease phosphate influx into the organism to avoid phosphaturia. The method for reducing the phosphate load and FGF23 levels was recently summarized (14).

The reduction of urinary citrate excretion and, by inference, the proximal tubule luminal citrate concentration, is a very early event in CKD. Importantly, reducing urinary citrate excretion increases the risk of calcium phosphate precipitation and luminal crystallopathy (15). The proximal reclamation of filtered citrate leaves its luminal concentration in the end of the proximal tubule sufficiently low, such that when luminal phosphate concentrations increase, as occurs in CKD, there is a marked risk for calcium phosphate precipitation. In addition to treating systemic metabolic acidosis, which is generally believed to be detrimental, alkaline therapy can also enhance citraturia, which should theoretically reduce the risk of calcium phosphate precipitation. However, the benefits of citraturia should be cautiously balanced against alkaline urine $\mathrm{pH}$, which was shown by Shiizaki, Tsubouchi, and colleagues to acutely enhance calcium phosphate precipitation (3). Enhanced calcium phosphate precipitation is one theoretical aspect of alkali therapy that should be explored further. Conversely, acute acid loading with ammonium chloride appears to lower urine $\mathrm{pH}$ to ameliorate calcium phosphate precipitation. The findings in this study using calcium phosphate crystals as a readout are too preliminary and should not be extrapolated to the management of acid-base disturbances in CKD.

Another target will be the suppression of high FGF23 levels, with the belief that FGF23 promotes phosphaturia and intraluminal calcium phosphate crystals.
However, the possibility that FGF23 is toxic in its own right, beyond promoting phosphaturic toxicity, further justifies the goal of lowering FGF23 levels. This topic was not addressed in the study by the authors. Shiizaki, Tsubouchi, and co-authors have shown that alternative TLR4 pathways may be involved and suggest that conventional TLR4 signaling blockers are ineffective at preventing CKD (3).

\section{Summary}

The paradigm outlined by Shiizaki, Tsubouchi, and coworkers and led by Kuro-o explains the progression of CKD from a mineral viewpoint (3). Instead of systemic phosphotoxicity, one is dealing with renal tubular lumen toxicity. Instead of effects from high phosphate concentrations, the toxicity is a true crystallopathy. The cycle can potentially self-propagate to drive CKD progression on its own. This data set from Shiizaki, Tsubouchi, and colleagues (3) prompts one to not just follow serum phosphate concentrations but to devote efforts to estimating the exposure of the proximal tubule lumen to phosphate and to monitoring the downstream effects of phosphate crystallopathy. If this model holds true in human CKD, researchers can develop treatments to arrest these destructive pathways and identify intermediate biomarkers of proximal luminal phosphate crystallopathy to personalize therapy.

\section{Acknowledgments}

The author's research is supported by the NIH (R01 DK081423, R01 DK115703, P30 DK079328, R01 HL134373, R01 DK091392) and the Charles Y C Pak Foundation.

Address correspondence to: Orson W. Moe, Charles and Jane Pak Center for Mineral Metabolism and Clinical Research, University of Texas Southwestern Medical Center, 5323 Harry
Hines Blvd., Dallas, Texas 75390, USA. Phone: 214.648.7993; Email: orson. moe@utsouthwestern.edu.

1. Coresh J, et al. Prevalence of chronic kidney disease in the United States. JAMA. 2007;298(17):2038-2047.

2. Mulay SR, Anders HJ. Crystal nephropathies: mechanisms of crystal-induced kidney injury. Nat Rev Nephrol. 2017;13(4):226-240.

3. Shiizaki K, et al. Calcium phosphate microcrystals in the renal tubular fluid accelerate chronic kidney disease progression. J Clin Invest. 2021; 145693 .

4. Heiss A, et al. Structural basis of calcification inhibition by alpha 2-HS glycoprotein/fetuin-A. Formation of colloidal calciprotein particles. J Biol Chem. 2003;278(15):13333-13341.

5. Herrmann M, et al. Clearance of fetuin-A--containing calciprotein particles is mediated by scavenger receptor-A. Circ Res. 2012;111(5):575-584.

6. Bricker NS, et al. The pathologic physiology of chronic Bright's disease. An exposition of the "intact nephron hypothesis". Am J Med. 1960;28:77-98.

7. Bricker NS. On the meaning of the intact nephron hypothesis. Am JMed. 1969;46(1):1-11.

8. Hostetter TH, et al. Hyperfiltration in remnant nephrons: a potentially adverse response to renal ablation. Am J Physiol. 1981;241(1):F85-F93.

9. Brenner BM, et al. The hyperfiltration theory: a paradigm shift in nephrology. Kidney Int. 1996;49(6):1774-1777.

10. Oliver J, Luey AB. Plastic studies in abnormal renal architecture. Arch Pathol. 1935;19:1-23.

11. Mackay EM, Oliver J. Renal damage following the ingestion of a diet containing an excess of inorganic phosphate. J Exp Med.1935;61(3):319-334

12. Marcussen N. Tubulointerstitial damage leads to atubular glomeruli: significance and possible role in progression. Nephrol Dial Transplant. 2000;15(Suppl 6):74-75.

13. Chevalier RL, Forbes MS. Generation and evolution of atubular glomeruli in the progression of renal disorders. JAm Soc Nephrol. 2008;19(2):197-206.

14. Verbueken D, Moe OW. Strategies to lower fibroblast growth factor-23 bioactivity [published online January 27, 2021]. Nephrol Dial Transplant. https://doi.org/10.1093/ndt/gfab012.

15. Gianella FG, et al. Spot urinary citrate-to-creatinine ratio is a marker for acid-base status in chronic kidney disease. Kidney Int . 2021;99(1):208-217. 
